\title{
Evaluation of the effectiveness of representative methods for determining Young's modulus and hardness from instrumented indentation data
}

\author{
Dejun Ma \\ Department of Mechanical Engineering, The Academy of Armored Forces Engineering, \\ Beijing 100072, People's Republic of China \\ Taihua Zhang \\ State Key Laboratory of Nonlinear Mechanics (LNM), Institute of Mechanics, Chinese Academy of \\ Sciences, Beijing 100080, People's Republic of China \\ Chung Wo Ong ${ }^{\text {a) }}$ \\ Department of Applied Physics and Materials Research Center, The Hong Kong Polytechnic \\ University, Hung Hom, Kowloon, Hong Kong, People's Republic of China
}

(Received 13 June 2005; accepted 3 October 2005)

\begin{abstract}
The effectiveness of Oliver \& Pharr's (O\&P's) method, Cheng \& Cheng's (C\&C's) method, and a new method developed by our group for estimating Young's modulus and hardness based on instrumented indentation was evaluated for the case of yield stress to reduced Young's modulus ratio $\left(\sigma_{\mathrm{y}} / E_{\mathrm{r}}\right) \geqslant 4.55 \times 10^{-4}$ and hardening coefficient $(n) \leqslant 0.45$. Dimensional theorem and finite element simulations were applied to produce reference results for this purpose. Both O\&P's and C\&C's methods overestimated the Young's modulus under some conditions, whereas the error can be controlled within $\pm 16 \%$ if the formulation was modified with appropriate correction functions. Similar modification was not introduced to our method for determining Young's modulus, while the maximum error of results was around $\pm 13 \%$. The errors of hardness values obtained from all the three methods could be even larger and were irreducible with any correction scheme. It is therefore suggested that when hardness values of different materials are concerned, relative comparison of the data obtained from a single standard measurement technique would be more practically useful. It is noted that the ranges of error derived from the analysis could be different if different ranges of material parameters $\sigma_{\mathrm{y}} / E_{\mathrm{r}}$ and $n$ are considered.
\end{abstract}

\section{INTRODUCTION}

Instrumented indentation was primarily invented for measuring elastic modulus and hardness of solids. ${ }^{1,2}$ An analysis normally starts from recording load and displacement data, from which the loading and unloading curves are drawn. This step can be accomplished accurately with the use of many commercial instrumented indentation systems. Obviously, the shape of the curves should reflect the deformation process, and they are expected to carry the information on the mechanical properties of the indented material. However, some key parameters required for calculating the Young's modulus and hardness of the tested material are not directly available from the raw data. One example is the contact depth

\footnotetext{
a) Address all correspondence to this author. e-mail: apacwong@inet.polyu.edu.hk DOI: $10.1557 / J M R .2006 .0019$
}

(or contact area) required for calculating the hardness value based on its primary definition. For this reason, a theoretical model is usually applied to establish some analytic formulation to correlate the apparent features of the loading and unloading curves with those parameters required. Since a theory is hardly ideal to suit all real cases, the methods developed from this route of thought are supposed to have various degrees of uncertainty. Based on this consideration, we suppose that there is a need to evaluate the effectiveness of some most popularly used methods in this area of study.

This article reports the evaluation of the effectiveness of three representative methods, i.e., Oliver \& Pharr's (O\&P's) method, ${ }^{3-5}$ Cheng \& Cheng's (C\&C's) method, ${ }^{6-10}$ and a method recently developed by our group. ${ }^{11-13}$ Section II summarizes the formulations associated with these approaches. Section III describes the details of the finite element analysis carried out in this study for simulating indentation processes made on 
hypothetical materials of different mechanical properties. In Secs. IV and V, the numerical results obtained from the finite element analysis are referred to for evaluating the effectiveness of the three methods. It was found that the Young's modulus and hardness values deduced from the three methods could deviate markedly from the reference values under some conditions. A remedial scheme was proposed to diminish the error in estimating Young's modulus. Similar observations and discussion were conducted for the investigations of the hardness value.

\section{SUMMARY OF REPRESENTATIVE METHODS}

According to O\&P's method, ${ }^{3-5}$ reduced Young's modulus $E_{\mathrm{r}}$, and hardness $H$ are expressed as follows

$$
\begin{gathered}
E_{\mathrm{r}}=\frac{\sqrt{\pi}}{2 \beta} \frac{S_{\mathrm{u}}}{\sqrt{A\left(h_{\mathrm{cm}}\right)}}, \\
H=P_{\mathrm{m}} / A\left(h_{\mathrm{cm}}\right),
\end{gathered}
$$

where $P_{\mathrm{m}}$ is the peak load, $h_{\mathrm{cm}}$ and $S_{\mathrm{u}}$ are the maximum contact depth and initial unloading stiffness at $P_{\mathrm{m}}, A\left(h_{\mathrm{cm}}\right)$ is the projected contact area measured at $h_{\mathrm{cm}}$, and $\beta$ an indenter geometry shape factor. Young's modulus $E$ of a material is calculated from $1 / E_{\mathrm{r}}=\left(1-v^{2}\right) /$ $E+\left(1-v_{\mathrm{i}}^{2}\right) / E_{\mathrm{i}}$, where $v$ and $v_{\mathrm{i}}$ are the Poisson's ratios of the indented material and indenter, and $E_{\mathrm{i}}$ is the elastic modulus of the indenter.

Depth-sensing indentation does not directly give the results of $h_{\mathrm{cm}}$ and $A\left(h_{\mathrm{cm}}\right)$, though they are required for calculating $E_{\mathrm{r}}$ and $H$ according to Eqs. (1) and (2). Sneddon suggested that $h_{\mathrm{cm}}$ should be associated with elastic deformation at the peak load $\eta P_{\mathrm{m}} / S_{\mathrm{u}}$ with $\eta=0.75$ for paraboloid revolution indenter, such that ${ }^{3,14}$

$$
h_{\mathrm{cm}}=h_{\mathrm{m}}-0.75 P_{\mathrm{m}} / S_{\mathrm{u}},
$$

where $S_{\mathrm{u}}$ is the initial slope of the unloading curve. The value of $h_{\mathrm{cm}}$ obtained in this way is just an indirect estimate, whereas any error induced would eventually propagate to the final results of $E$ and $H$.

In C\&C's method, the work done associated with the recovery of elastic deformation during unloading $W_{\mathrm{e}}$, and the total work done during loading $W$ are calculated from the areas under the unloading and loading curves. ${ }^{6,7}$ The ratio $W_{\mathrm{e}} / W$ is found to correlate with $H / E_{\mathrm{r}}$ through a functional relationship, which can be expressed in an implicit form of

$$
H / E_{\mathrm{r}}=f\left(W_{\mathrm{e}} / W\right)
$$

The discovery of this new relationship eliminates the need of knowing $h_{\mathrm{cm}}$ as in O\&P's method. A set of formulation is then established by eliminating $A\left(h_{\mathrm{cm}}\right)$ from Eqs. (1) and (2), and combining with Eq. (4) to solve for $E_{\mathrm{r}}$ and $H$, where $A\left(h_{\mathrm{cm}}\right)$ does not appear:

$$
\begin{gathered}
E_{\mathrm{r}}=\frac{\pi}{4 \beta^{2}} f\left(W_{\mathrm{e}} / W\right) \frac{S_{\mathrm{u}}{ }^{2}}{P_{\mathrm{m}}}, \\
H=\frac{\pi}{4 \beta^{2}}\left[f\left(W_{\mathrm{e}} / W\right)\right]^{2} \frac{S_{\mathrm{u}}{ }^{2}}{P_{\mathrm{m}}} .
\end{gathered}
$$

In another method recently developed by our group, ${ }^{11-13}$ a new quantity named "nominal hardness" was introduced, which was defined as $H_{\mathrm{n}} \equiv P_{\mathrm{m}} / A\left(h_{\mathrm{m}}\right)$. Its definition is fundamentally different from the conventional hardness $H$, since its denominator is the area measured at $h_{\mathrm{m}}$ but not $h_{\mathrm{cm}}$. The distinctive feature accompanying such a definition is that $H_{\mathrm{n}}$ is an accurately determinable quantity, since both $P_{\mathrm{m}}$ and $h_{\mathrm{m}}$ are accurately measurable in a standard depth-sensing indentation test. Based on dimensional analysis and finite element analysis for ideally sharp conical indenter shape, it is found that a functional relationship between $H_{\mathrm{n}} / E_{\mathrm{r}}$ and $W_{\mathrm{e}} / W$ exists, and is expressible implicitly as

$$
H_{\mathrm{n}} / E_{\mathrm{r}}=\Phi\left(W_{\mathrm{e}} / W\right)
$$

The advantage of this approach is immediately seen, because $E_{\mathrm{r}}$ can now be expressed as

$$
E_{\mathrm{r}}=H_{\mathrm{n}} / \Phi\left(W_{\mathrm{e}} / W\right)
$$

The parameters related to the right hand side (i.e., $P_{\mathrm{m}}$, $h_{\mathrm{m}}, W_{\mathrm{e}}$, and $W$ ) are all accurately measurable in a standard indentation test. Provided that the explicit form of $\Phi\left(W_{\mathrm{e}} / W\right)$ can be derived by some means, such as precision finite element simulation, in principle the value of $E_{\mathrm{r}}$ determined from Eq. (8) would more truly reflect the actual value. Similar comments are also applicable to the determination of conventional hardness, which expression is obtained by combining Eqs. (4) and (7):

$$
H=\left[\frac{f\left(W_{\mathrm{e}} / W\right)}{\Phi\left(W_{\mathrm{e}} / W\right)}\right] H_{\mathrm{n}} \quad .
$$

\section{FINITE ELEMENT SIMULATION OF INSTRUMENTED INDENTATION}

Numerical simulations of mechanical response in hypothetical indentation tests were conducted. The purpose was to produce reference values to be used in evaluating the effectiveness of the three said methods. A commercial finite element code ABAQUS ${ }^{15}$ was used. The package is well-known of its spectacular ability in handling problems of large deformation and strong nonlinearity. Calculations were performed within the framework of continuum mechanics. In particular, in the finite element simulations, four-node axisymmetric elements are used, and the size of the elements in contact with the indenter 
is small enough to ensure that at least 30 nodes are in contact with the indenter. The indenter was assigned to have an ideally sharp conical shape with a half-included angle of $70.3^{\circ}$, which gave the same area-to-depth ratio as that of the ideal Berkovich geometry.

The indented material was assumed to behave as an isotropic and rate-independent solid, obeying the Von Mises yield criterion and pure isotropic hardening rule. The uniaxial stress-strain relation is assumed to be a combination of linear elasticity and Hollomon's power law hardening in the form of

$$
\sigma=\left\{\begin{array}{ll}
E \epsilon & , \epsilon \leqslant \epsilon_{\mathrm{y}} \\
\sigma_{\mathrm{y}}\left(\epsilon / \epsilon_{\mathrm{y}}\right)^{n} & , \epsilon>\epsilon_{\mathrm{y}}
\end{array},\right.
$$

$\sigma$ and $\epsilon$ are the true stress and true strain; and $\sigma_{\mathrm{y}}$ and $\epsilon_{\mathrm{y}}=\sigma_{\mathrm{y}} / E$ where the yield stress and yield strain. The contact interface was assumed to be frictionless.

We first prove an assertion which will be repetitively used in further analysis. It states that an arbitrary dimensionless quantity related to an indentation test can be expressed as a function of $\sigma_{\mathrm{y}} / E_{\mathrm{r}}$ and $n$, or alternatively as a function of $W_{\mathrm{e}} / W$ and $n$. At present, we designate such a quantity as $Q$, which could be used to refer to $\sigma_{\mathrm{y}} / E_{\mathrm{r}}$, $W_{\mathrm{e}} / W, H / E_{\mathrm{r}}, H_{\mathrm{n}} / E_{\mathrm{r}}$, and so on. To prove the assertion, one acknowledges that any $Q$ must be a function of the elastoplastic properties $\left(E, \nu, \sigma_{\mathrm{y}}, n\right)$ of the indented material, the elastic properties $\left(E_{\mathrm{i}}, v_{\mathrm{i}}\right)$ of the indenter and maximum displacement $h_{\mathrm{m}}$. Therefore, it can be expressed implicitly as

$$
Q=f_{Q}\left(E, \nu, \sigma_{\mathrm{y}}, n, E_{\mathrm{i}}, \nu_{\mathrm{i}}, h_{\mathrm{m}}\right) .
$$

According to Dao et al. ${ }^{16}$ the number of independent variables can be reduced by introducing $E_{\mathrm{r}}$, which combines all the elasticity effects from the indenter and indented material. As such, Eq. (11) is simplified as:

$$
Q=f_{Q}\left(\sigma_{\mathrm{y}}, n, E_{\mathrm{r}}, h_{\mathrm{m}}\right) \text {. }
$$

By applying the $\Pi$ theorem of dimensional analysis, two more independent variables can be removed. Consequently, the implicit expression of $Q$ is further simplified as

$$
Q=\Psi_{Q}\left(\sigma_{\mathrm{y}} / E_{\mathrm{r}}, n\right) .
$$

Considering that $W_{\mathrm{e}} / W$ is a dimensionless quantity, Eq. (13) is also applicable to $W_{\mathrm{e}} / W$ :

$$
W_{\mathrm{e}} / W=\Psi_{W}\left(\sigma_{\mathrm{y}} / E_{\mathrm{r}}, n\right) .
$$

Alternatively, $\sigma_{\mathrm{y}} / E_{\mathrm{r}}$ can be expressed in terms of $W_{\mathrm{e}} / W$ and $n$ :

$$
\sigma_{\mathrm{y}} / E_{\mathrm{r}}=\Psi_{W 1}\left(W_{\mathrm{e}} / W, n\right)
$$

By substituting Eq. (15) into Eq. (13) to remove $\sigma_{\mathrm{y}} / E_{\mathrm{r}}$, the expression of $Q$ becomes

$$
Q=\Psi_{Q}\left[\Psi_{W 1}\left(W_{\mathrm{e}} / W, n\right), n\right]=\Gamma_{Q}\left(W_{\mathrm{e}} / W, n\right) .
$$

The above steps only illustrate the existence of the Eqs. (11)-(16). In practice, explicit relationships are required in real analysis and must be derived in detail by some means. The first explicit function required in this study is the numerical dependence of $W_{\mathrm{e}} / W$ on $\sigma_{\mathrm{y}} / E_{\mathrm{r}}$ and $n$, namely Eq. (14). Some more will be required and introduced in subsequent parts of discussions. We applied finite element simulations to produce the explicit forms of these functions, with $\sigma_{\mathrm{y}} / E_{\mathrm{r}}$ and $n$ being the parameters to vary in broad ranges. Consider the dimensionless ratio $\sigma_{\mathrm{y}} / E_{\mathrm{r}}$ first. Its value is conveniently varied by scanning $\sigma_{\mathrm{y}}$ over some range with $E_{\mathrm{r}}$ fixed at a certain value. Recalling that $1 / E_{\mathrm{r}}=\left(1-v^{2}\right) / E+\left(1-v_{\mathrm{i}}^{2}\right) / E_{\mathrm{i}}$, the two parameters $E_{\mathrm{i}}$ and $\nu_{\mathrm{i}}$ can be dropped first by assuming that the indenter is rigid. By setting $E=70 \mathrm{GPa}$, $v=0.3$, and $h_{\mathrm{m}}=1 \mu \mathrm{m}$, the value of $E_{\mathrm{r}}$ was specified to be $E /\left(1-v^{2}\right)=76.923 \mathrm{GPa}$. Thereafter, this value is denoted as $E_{\mathrm{r}}^{\mathrm{t}}$ and is referred to as the "true" reduced Young's modulus (more other reference values will be introduced subsequently for the purpose of comparing with the corresponding calculated results, and they will be designated by a superscript $t$ ). It is used to compare with the $E_{\mathrm{r}}$ values deduced from Eqs. (1), (5), and (8) to evaluate the effectiveness of the three methods concerned. The setting of its absolute value would not affect the conclusion of the evaluation. $\sigma_{\mathrm{y}}$ was assigned by one of the fourteen values of $0.035,0.140,0.350,0.700$, $1.400,2.100,2.800,3.500,4.550,5.600,7.000,10.500$, 14.000 , and $17.500 \mathrm{GPa} . n$ is assigned by one of the four values, namely $0,0.15,0.3$, and 0.45 . As such, there were totally 56 different combinations of the two parameters in the calculations. The detailed features of the mesh are described elsewhere. ${ }^{11,17}$ A sensitivity study proceeded by reducing the mesh size by one half showed that the calculated peak load would not deviate from the original result by more than $0.6 \%$, confirming that the original mesh size adequately models the hypothetical conical indentation on semi-infinite solid.

\section{EVALUATION OF THE THREE METHODS USED FOR ESTIMATING YOUNG'S MODULUS}

\section{A. O\&P's method}

We first inspect the results of $E_{\mathrm{r}}$ deduced from O\&P's method. For a certain combination of $\sigma_{\mathrm{y}}$ and $n$, a set of loading and unloading curves was produced from numerical simulation, and hence the values of $P_{\mathrm{m}}$ and $S_{\mathrm{u}}$ were determined. They were used to estimate $h_{\mathrm{cm}}$ from Eq. (3), meanwhile, $\beta$ was set to be 1.096 according to Dao et al.'s suggestion. ${ }^{16}$ The contact area for an ideally sharp conical indenter with a half-included angle of $70.3^{\circ}$ 
was calculated to be $A\left(h_{\mathrm{cm}}\right)=24.5 h_{\mathrm{cm}}{ }^{2}$. The value of $E_{\mathrm{r}}$ was then estimated from Eq. (1). A dimensionless quantity $Q_{1} \equiv E_{\mathrm{r}} / E_{\mathrm{r}}{ }^{\mathrm{t}}$ was thus defined, and according to Eq. (16), it should be expressible with a function $\Gamma_{Q 1}\left(W_{\mathrm{e}} / W, n\right)$

$$
Q_{1}=\left[\frac{\sqrt{\pi}}{2 \beta} \frac{S_{\mathrm{u}}}{\sqrt{A\left(h_{\mathrm{cm}}\right)}}\right] / E_{\mathrm{r}}^{\mathrm{t}}=\Gamma_{Q 1}\left(W_{\mathrm{e}} / W, n\right)
$$

If O\&P's method works properly for all materials, $Q_{1}$ should be constantly close to 1 disregarding the variation in $\sigma_{\mathrm{y}}$ and $n$ [or equivalently $W_{\mathrm{e}} / W$ and $n$ according to Eq. (14)]. Figure 1 shows how the results of $E_{\mathrm{r}} / E_{\mathrm{r}}{ }_{\mathrm{r}}^{\mathrm{t}}$ depend on different combinations of $W_{\mathrm{e}} / W$ and $n$. In the region of $W_{\mathrm{e}} / W<0.25, Q_{1}$ is obviously larger than 1 , except some points of $n=0.45$. This indicates that in low $W_{\mathrm{e}} / W$ region, $E_{\mathrm{r}}$ tends to be overestimated by O\&P's method.

One possible reason for the overestimation of $E_{\mathrm{r}}$ is that $h_{\mathrm{cm}}$ [hence $\left.A\left(h_{\mathrm{cm}}\right)\right]$ is underestimated. To elucidate this possibility, we refer to another dimensionless ratio $Q_{2} \equiv h_{\mathrm{cm}} / h_{\mathrm{cm}}{ }^{\mathrm{t}}$, where $h_{\mathrm{cm}}{ }^{\mathrm{t}}$ is the "true" contact depth determined from the simulations. According to Eq. (16), $h_{\mathrm{cm}} / h_{\mathrm{cm}}{ }^{\mathrm{t}}$ can be expressed by a function $\Gamma_{Q 2}\left(W_{\mathrm{e}} / W, n\right)$, where

$$
Q_{2}=\left[h_{\mathrm{m}}-0.75 P_{\mathrm{m}} / S_{\mathrm{u}}\right] / h_{\mathrm{cm}}{ }^{\mathrm{t}}=\Gamma_{Q 2}\left(W_{\mathrm{e}} / W, n\right)
$$

Figure 2 shows that at low $W_{\mathrm{e}} / W$ region, $\Gamma_{Q 2}\left(W_{\mathrm{e}} / W, n\right)$ are lower than 1, except some data points of $n=0.45$. This observation is consistent with the results of some previous studies. ${ }^{18-20}$ Another possible reason for the overestimation of $E_{\mathrm{r}}$ is that the indenter shape factor $\beta$ in Eq. (1) is a variable but not a constant. Its value is underestimated in low $W_{\mathrm{e}} / W$ region. If this conjecture is correct, the "true" value of $\beta$ (denoted as $\beta^{t}$ ) can be

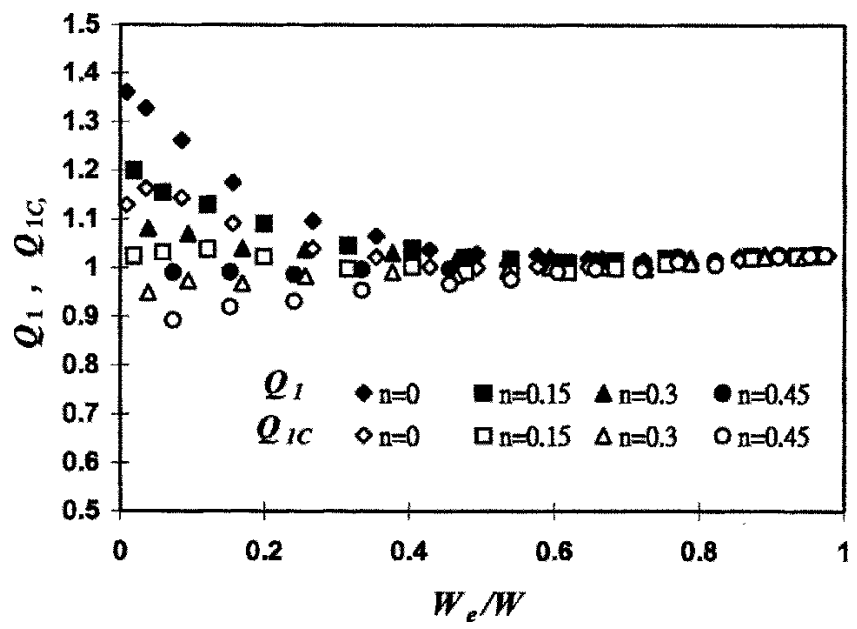

FIG. 1. Plots of numerical results of $\Gamma_{Q 1}\left(W_{\mathrm{e}} / W, n\right) \equiv E_{\mathrm{r}} / E_{\mathrm{r}}$; and $\Gamma_{Q 1 C}$ $\left(W_{\mathrm{e}} / W, n\right) \equiv E_{\mathrm{rC}} / E_{\mathrm{r}}^{\mathrm{t}}$ obtained from O\&P's method.

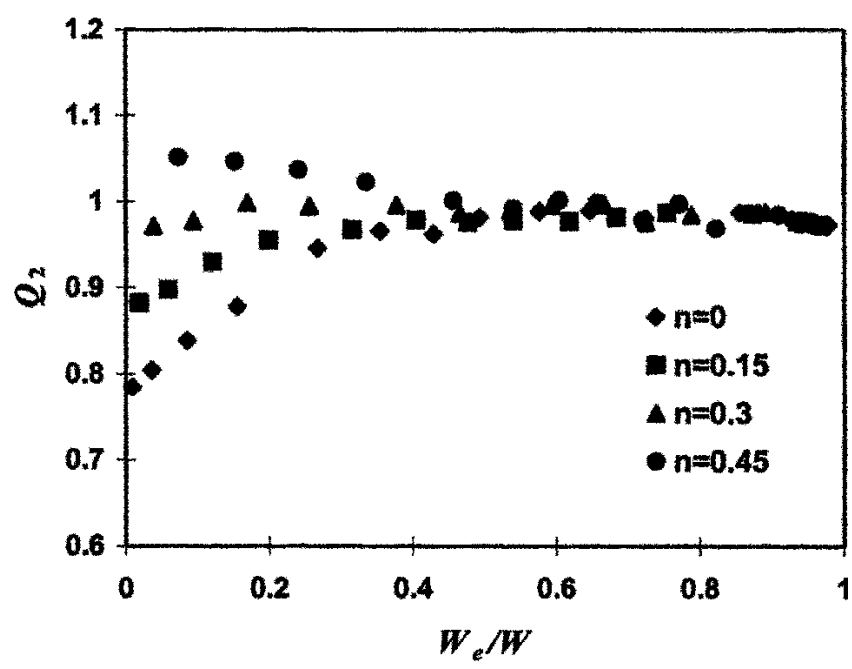

FIG. 2. Plot of numerical results of $\Gamma_{Q 2}\left(W_{\mathrm{e}} / W, n\right) \equiv h_{\mathrm{cm}} / h_{\mathrm{cm}}{ }^{\mathrm{t}}$ obtained from O\&P's method.

attained by replacing $h_{\mathrm{cm}}$ and $E_{\mathrm{r}}$ with $h_{\mathrm{cm}}{ }^{\mathrm{t}}$ and $E_{\mathrm{r}}^{\mathrm{t}}$ in Eq. (1), such that

$$
\beta^{\mathrm{t}}=\left[\frac{\sqrt{\pi}}{2} \frac{S_{\mathrm{u}}}{\sqrt{A\left(h_{\mathrm{cm}}^{\mathrm{t}}\right)}}\right] / E_{\mathrm{r}}^{\mathrm{t}} .
$$

Being a dimensionless quantity, Eq. (16) is applicable to $\beta^{\mathrm{t}}$, expecting that it is equal to a function like $\Gamma_{\beta}$ $\left(W_{\mathrm{e}} / W, n\right)$

$$
\beta^{\mathrm{t}}=\left[\frac{\sqrt{\pi}}{2} \frac{S_{\mathrm{u}}}{\sqrt{A\left(h_{\mathrm{cm}}^{\mathrm{t}}\right)}}\right] / E_{\mathrm{r}}^{\mathrm{t}}=\Gamma_{\beta}\left(W_{\mathrm{e}} / W, n\right) .
$$

Figure 3 shows the numerical results of $\Gamma_{\beta}\left(W_{\mathrm{e}} / W, n\right)$ for different combinations of $W_{\mathrm{e}} / W$ and $n$. As expected, $\beta^{\mathrm{t}}$ behaves as a variable rather than a constant. It deviates

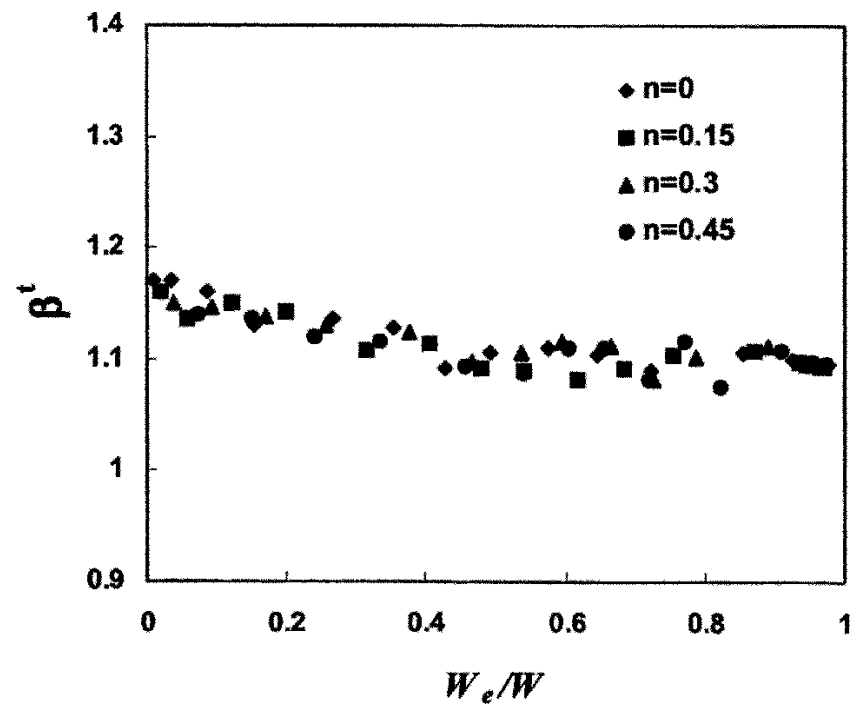

FIG. 3. Plot of numerical results of $\Gamma_{\beta}\left(W_{\mathrm{e}} / W, n\right) \equiv \beta^{\mathrm{t}}$. 
more markedly from the preset value 1.096 when $W_{\mathrm{e}} / W<0.25$.

The error in estimating $E_{\mathrm{r}}$ is considered to be suppressible by multiplying the following correction function to Eq. (1):

$$
F_{\mathrm{c}}\left(W_{\mathrm{e}} / W\right)=(\beta / 1.096)\left(W_{\mathrm{e}} / W\right)^{0.04} .
$$

The expressions of $E_{\mathrm{r}}$ and $Q_{1}$ are thus modified and denoted as $E_{\mathrm{rC}}$ and $Q_{1 \mathrm{C}}=E_{\mathrm{rC}} / E_{\mathrm{r}}^{\mathrm{t}}$ thereafter, which can be written as:

$$
\begin{aligned}
E_{\mathrm{rC}} & =\left[\frac{\sqrt{\pi}}{2 \beta} \frac{S_{\mathrm{u}}}{\sqrt{A\left(h_{\mathrm{cm}}\right)}}\right]\left(\frac{\beta}{1.096}\right)\left(W_{\mathrm{e}} / W\right)^{0.04} \\
& =\left[\frac{\sqrt{\pi}}{2.192} \frac{S_{\mathrm{u}}}{\sqrt{A\left(h_{\mathrm{cm}}\right)}}\right]\left(W_{\mathrm{e}} / W\right)^{0.04}, \\
Q_{1 \mathrm{C}} & =\left[\frac{\sqrt{\pi}}{2.192} \frac{S_{\mathrm{u}}}{\sqrt{A\left(h_{\mathrm{cm}}\right)}}\right]\left(W_{\mathrm{e}} / W\right)^{0.04} / E_{\mathrm{r}}^{\mathrm{t}} \\
& =\Gamma_{Q 1 \mathrm{C}}\left(W_{\mathrm{e}} / W, n\right) .
\end{aligned}
$$

We note that other values of $\beta$ in Eq. (1) may be used by different authors, but according to our correction scheme, after multiplying $F_{\mathrm{c}}\left(W_{\mathrm{e}} / W\right)$ to Eq. (1), the factor $\beta$ is cancelled and would not appear in Eqs. (22) and (23). Instead, only a constant factor of 1.096 is seen in the equations. Figure 1 shows the calculated values of $Q_{1 \mathrm{C}}$, which indicate that the error band is confined within $\pm 16 \%$ after our correction scheme is used.

\section{B. C\&C's method}

Applying Eq. (16) again, the dimensionless quantity $H / E_{\mathrm{r}}$ is expressible as a function like $\Gamma_{\mathrm{H}}\left(W_{\mathrm{e}} / W, n\right)$ :

$$
H / E_{\mathrm{r}}=\Gamma_{\mathrm{H}}\left(W_{\mathrm{e}} / W, n\right) .
$$

Figure 4(a) shows the explicit form of $\Gamma_{\mathrm{H}}\left(W_{\mathrm{e}} / W, n\right)$ obtained from hypothetical simulations. Details in the range of $W_{\mathrm{e}} / W \leqslant 0.3$ are exaggerated and shown in Fig. 4(b). The presence of a function form between $H / E_{\mathrm{r}}$ and $W_{\mathrm{e}} / W$ is strongly evident, which is regressed by a polynomial of

$$
H / E_{\mathrm{r}}=f\left(W_{\mathrm{e}} / W\right)=\sum_{i=1}^{6} a_{\mathrm{i}}\left(W_{\mathrm{e}} / W\right)^{\mathrm{i}} .
$$

The coefficients of the terms are determined to be $a_{1}=$ $0.14736, a_{2}=0.15960, a_{3}=-0.23052, a_{4}=0.12656$, $a_{5}=0.18514$, and $a_{6}=-0.19733$.

Let $Q_{3} \equiv E_{\mathrm{r}} / E_{\mathrm{r}}^{\mathrm{t}}$ to be equal to the right side of Eq. (5) divided by $E_{\mathrm{r}}^{\mathrm{t}}$. It is also dimensionless and so can be expressed as a function like $\Gamma_{Q 3}\left(W_{\mathrm{e}} / W, n\right)$ :
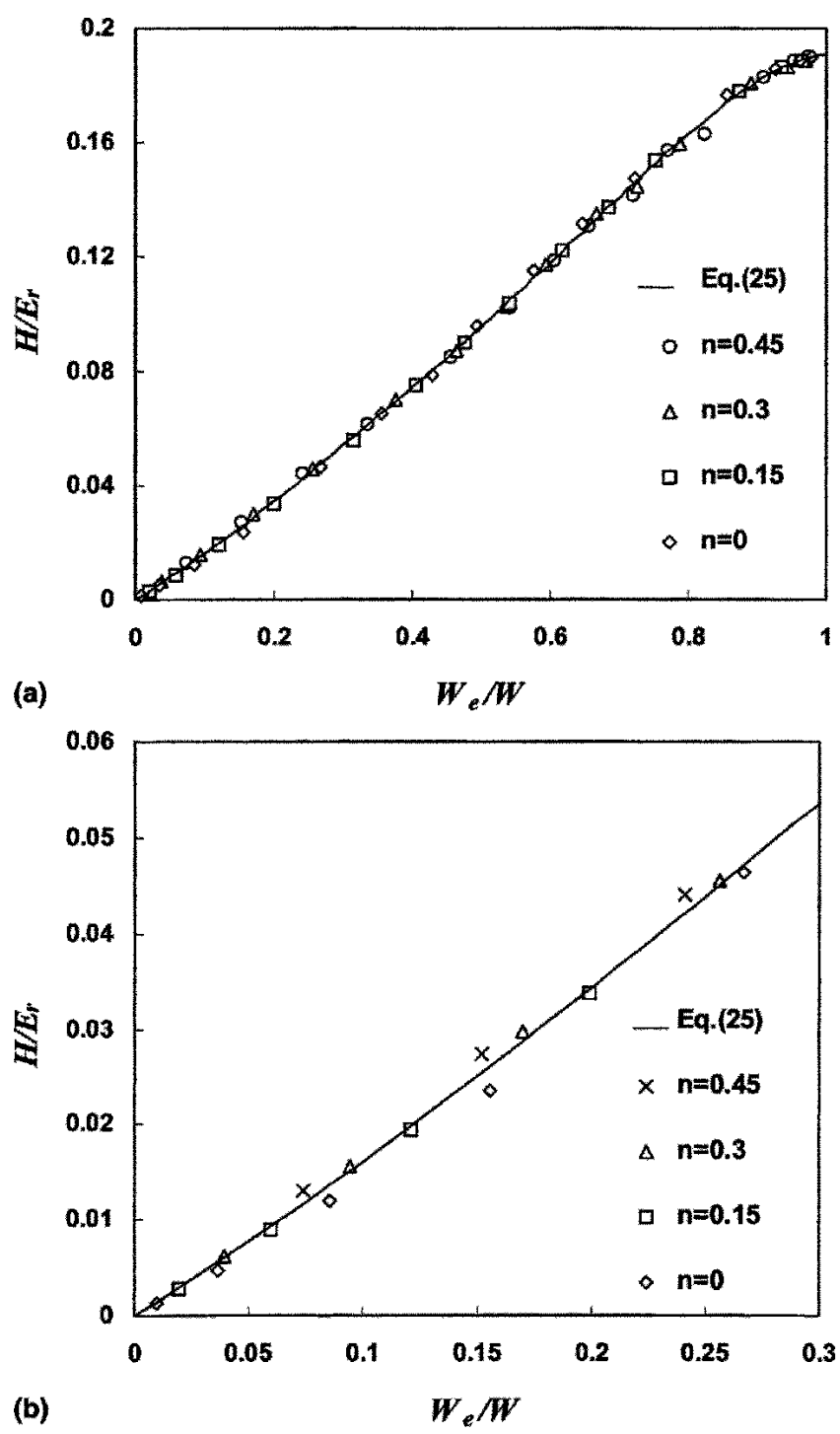

FIG. 4. (a) Functional relationship between $H / E_{\mathrm{r}}$ and $W_{\mathrm{e}} / W$ and (b) exaggeration in the range of $W_{\mathrm{e}} / W \leqslant 0.3$ used in C\&C's method.

$$
Q_{3}=\left[\frac{\pi}{4 \beta^{2}} f\left(W_{\mathrm{e}} / W\right) \frac{S_{\mathrm{u}}^{2}}{P_{\mathrm{m}}}\right] / E_{\mathrm{r}}^{\mathrm{t}}=\Gamma_{Q 3}\left(W_{\mathrm{e}} / W, n\right)
$$

Figure 5 shows the numerical results of $\Gamma_{Q 3}\left(W_{\mathrm{e}} / W, n\right)$. In the region of $W_{\mathrm{e}} / W$ below 0.25 , most of the data points are higher than 1 , except those of $n=0.45$. This indicates that the deduced $E_{\mathrm{r}}$ values are overestimated in this region. One reason of the overestimation is that the indenter shape factor $\beta$ is assumed to be a constant in the calculations, but it could actually be a variable increasing with decreasing $W_{\mathrm{e}} / W$. If so, the error would be magnified in the form of $\beta^{2}$ as shown in Eq. (26). The ultimate cause of the error may be based on the fact that the functional relationship Eq. (25) is just approximately correct but not reflecting all the features of the real case.

The error in estimating $E_{\mathrm{r}}$ with C\&C's method can be 


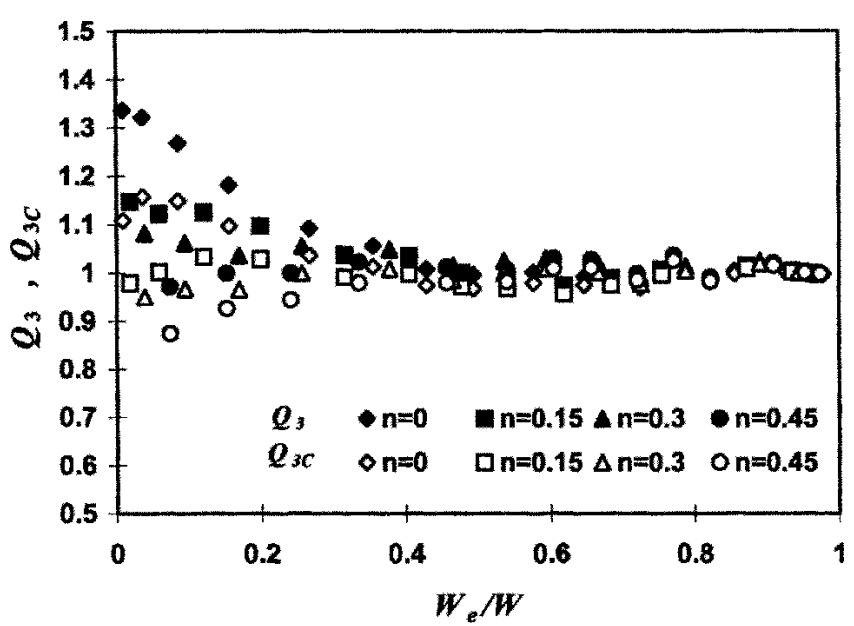

FIG. 5. Plots of numerical results of $\Gamma_{Q 3}\left(W_{\mathrm{e}} / W, n\right) \equiv E_{\mathrm{r}} / E_{\mathrm{r}}^{\mathrm{t}}$; and $\Gamma_{Q 3 C}$ $\left(W_{\mathrm{e}} / W, n\right) \equiv E_{\mathrm{rC}} / E_{\mathrm{r}}^{\mathrm{t}}$ obtained from C\&C's method.

suppressed by multiplying a correction function to Eq. (5), which is suggested to have the form of

$$
F_{\mathrm{c}}\left(W_{\mathrm{e}} / W\right)=(\beta / 1.096)^{2}\left(W_{\mathrm{e}} / W\right)^{0.04}
$$

This procedure modifies Eqs. (5) and (26) to result in the following formulas:

$$
\begin{aligned}
E_{\mathrm{rC}} & =\left[\frac{\pi}{4 \beta^{2}} f\left(W_{\mathrm{e}} / W\right) \frac{S_{\mathrm{u}}^{2}}{P_{\mathrm{m}}}\right]\left(\frac{\beta}{S_{\mathrm{u}}^{2}}\right)^{2}\left(W_{\mathrm{e}} / W\right)^{0.04} \\
& =\left[\frac{\pi}{4.8049} f\left(W_{\mathrm{e}} / W\right) \frac{{ }_{\mathrm{u}}}{P_{\mathrm{m}}}\right]\left(W_{\mathrm{e}} / W\right)^{0.04}, \\
Q_{3 \mathrm{C}} & =\left[\frac{\pi}{4.8049} f\left(W_{\mathrm{e}} / W\right) \frac{S_{\mathrm{u}}^{2}}{P_{\mathrm{m}}}\right]\left(W_{\mathrm{e}} / W\right)^{0.04} / E_{\mathrm{r}}^{\mathrm{t}} \\
& =\Gamma_{Q 3 \mathrm{C}}\left(W_{\mathrm{e}} / W, n\right) .
\end{aligned}
$$

The data points of $Q_{3 \mathrm{C}}$ are shown in Fig. 5 to compare with $Q_{3}$, where the error band of the estimated reduced Young's modulus is found to fall within $\pm 16 \%$.

\section{Our method}

Since $H_{\mathrm{n}} / E_{\mathrm{r}}$ is a dimensionless quantity, so that from Eq. (16), it is ascertained that there must be a function $\Gamma_{\mathrm{Hn}}\left(W_{\mathrm{e}} / W, n\right)$ such that

$$
H_{\mathrm{n}} / E_{\mathrm{r}}=\Gamma_{\mathrm{Hn}}\left(W_{\mathrm{e}} / W, n\right)
$$

Figure 6(a) shows the numerical results of $\Gamma_{\mathrm{Hn}}\left(W_{\mathrm{e}} / W, n\right)$. Details in the region of $W_{\mathrm{e}} / W \leqslant 0.3$ is exaggerated in Fig. 6(b). From the figure, the functional dependence of $H_{\mathrm{n}} / E_{\mathrm{r}}$ on $W_{\mathrm{e}} / W$ is strongly evident. The data can be regressed by using a polynomial in the form of

$$
H_{\mathrm{n}} / E_{\mathrm{r}}=\Phi\left(W_{\mathrm{e}} / W\right)=\sum_{i=1}^{6} b_{\mathrm{i}}\left(W_{\mathrm{e}} / W\right)^{\mathrm{i}}
$$

The coefficients of the terms are determined to be

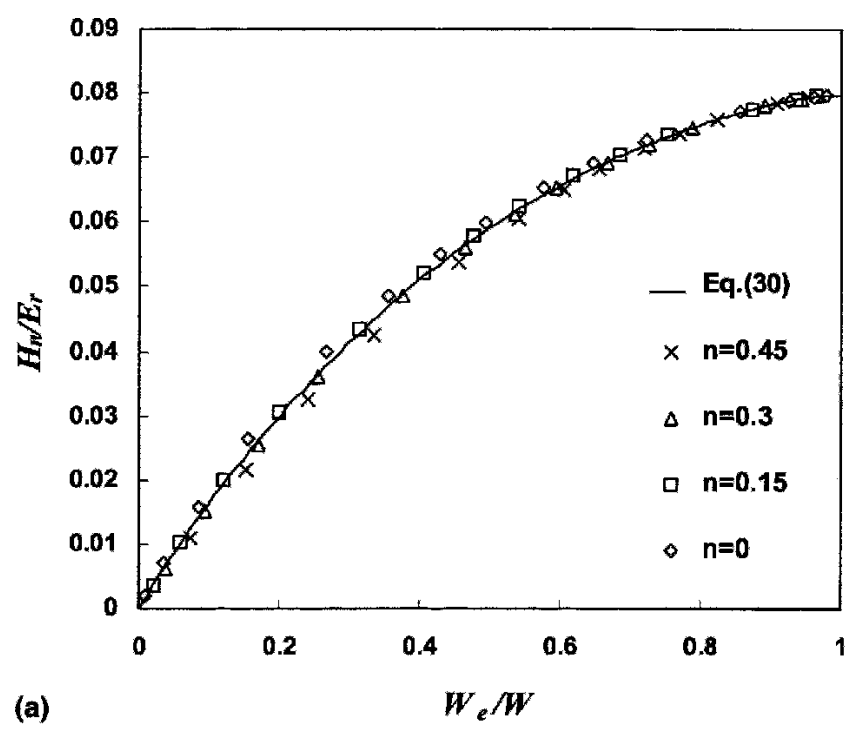

(a)

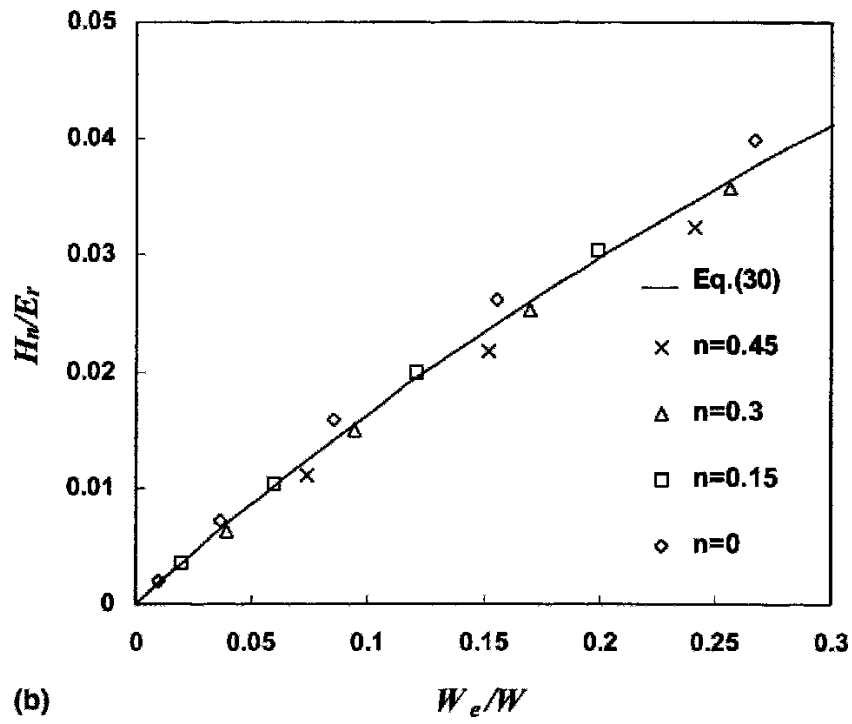

FIG. 6. (a) Functional relationship between $H_{\mathrm{n}} / E_{\mathrm{r}}$ and $W_{\mathrm{e}} / W$ and (b) exaggeration in the range of $W_{\mathrm{e}} / W \leqslant 0.3$ used in our method.

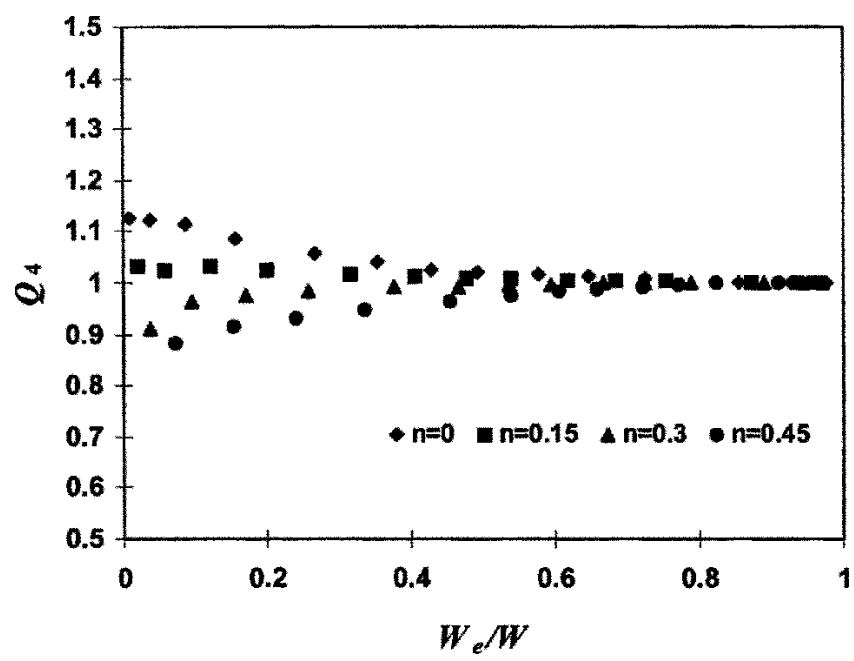

FIG. 7. Plot of numerical results of $\Gamma_{Q 4}\left(W_{\mathrm{e}} / W, n\right) \equiv E_{\mathrm{r}} / E_{\mathrm{r}}^{\mathrm{t}}$ obtained from our method. 
TABLE I. The $E_{\mathrm{r}} / E_{\mathrm{r}}^{\mathrm{t}}$ values estimated from the three methods by using the data given in Alkorta et al. ${ }^{22}$ Poisson's ratio is set to be 0.2 .

\begin{tabular}{|c|c|c|c|c|c|c|c|}
\hline$n$ & $E(\mathrm{MPa})$ & $E_{\mathrm{r}}(\mathrm{MPa})$ & $\sigma_{\mathrm{y}}(\mathrm{MPa})$ & $\sigma_{\mathrm{y}} / E_{\mathrm{r}}$ & $E_{\mathrm{r}} / E_{\mathrm{r}}^{\mathrm{t}}(\mathrm{O} \& \mathrm{P} ' \mathrm{~s})$ & $E_{\mathrm{r}} / E_{\mathrm{r}}^{\mathrm{t}}\left(\mathrm{C} \& \mathrm{C}^{\prime} \mathrm{s}\right)$ & $E_{\mathrm{r}} / E_{\mathrm{r}}^{\mathrm{t}}($ ours $)$ \\
\hline 0 & 10160 & 10583.33 & 10.16 & $9.60 \times 10^{-4}$ & 1.3718 & 1.3933 & 1.1130 \\
\hline 0.1 & 11072.36 & 11533.71 & 5.483678 & $4.7545 \times 10^{-4}$ & 1.2465 & 1.2172 & 1.0513 \\
\hline 0.2 & 11981.13 & 12480.34 & 2.974937 & $2.3837 \times 10^{-4}$ & 1.1805 & 1.1754 & 0.9763 \\
\hline 0.3 & 12767.38 & 13299.36 & 1.406929 & $1.0579 \times 10^{-4}$ & 1.1138 & 1.1201 & 0.9117 \\
\hline 0.4 & 13368.42 & 13925.44 & 0.551738 & $3.9621 \times 10^{-5}$ & 1.0237 & 1.0104 & 0.8538 \\
\hline
\end{tabular}

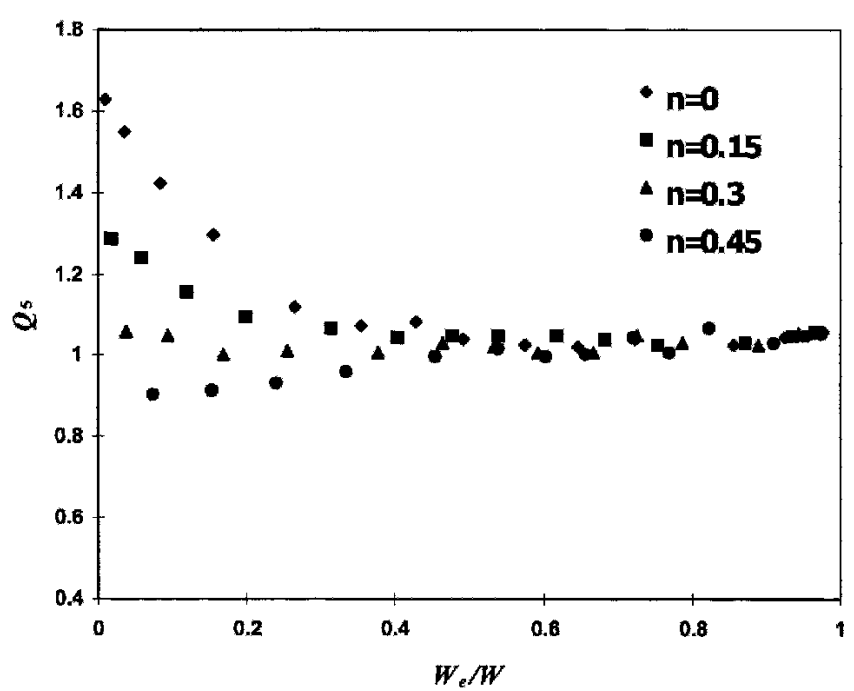

FIG. 8. Plot of numerical results of $\Gamma_{Q 5}\left(W_{\mathrm{e}} / W, n\right) \equiv H / H^{\mathrm{t}}$ obtained from O\&P's method.

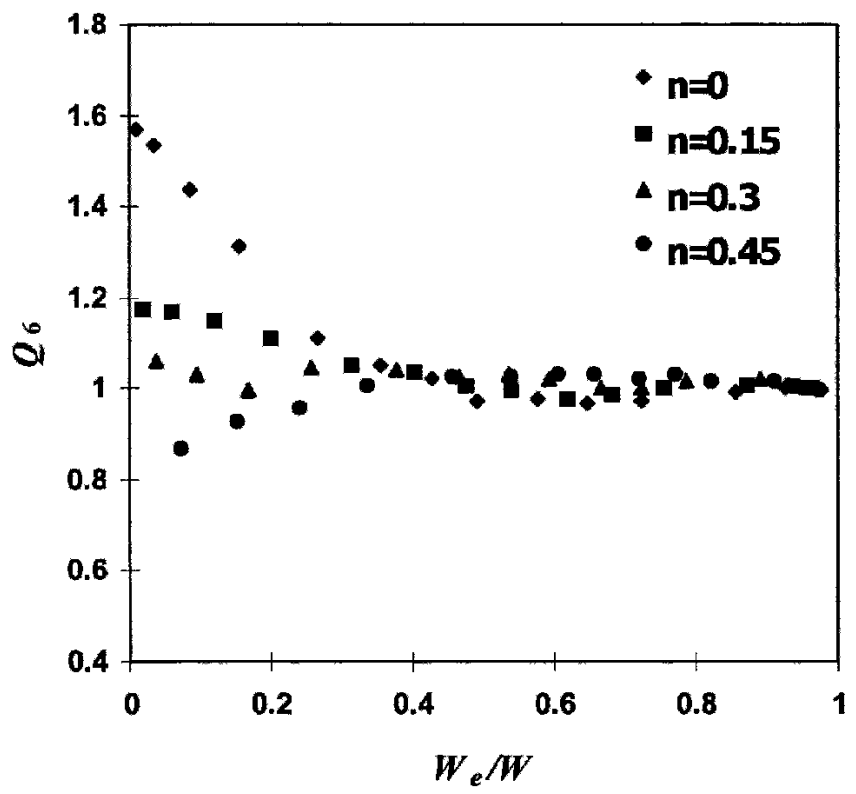

FIG. 9. Numerical results of function $\Gamma_{Q 6}\left(W_{\mathrm{e}} / W, n\right) \equiv H / H^{\mathrm{t}}$ obtained from C\&C's method.

$b_{1}=0.18408, b_{2}=-0.24835, b_{3}=0.50721$, $b_{4}=-0.86118, b_{5}=0.75187$, and $b_{6}=-0.25388$.

We compared the result of a special case of $W_{\mathrm{e}} / W=1$ with that reported by Hay et al. ${ }^{21}$ This corresponds to

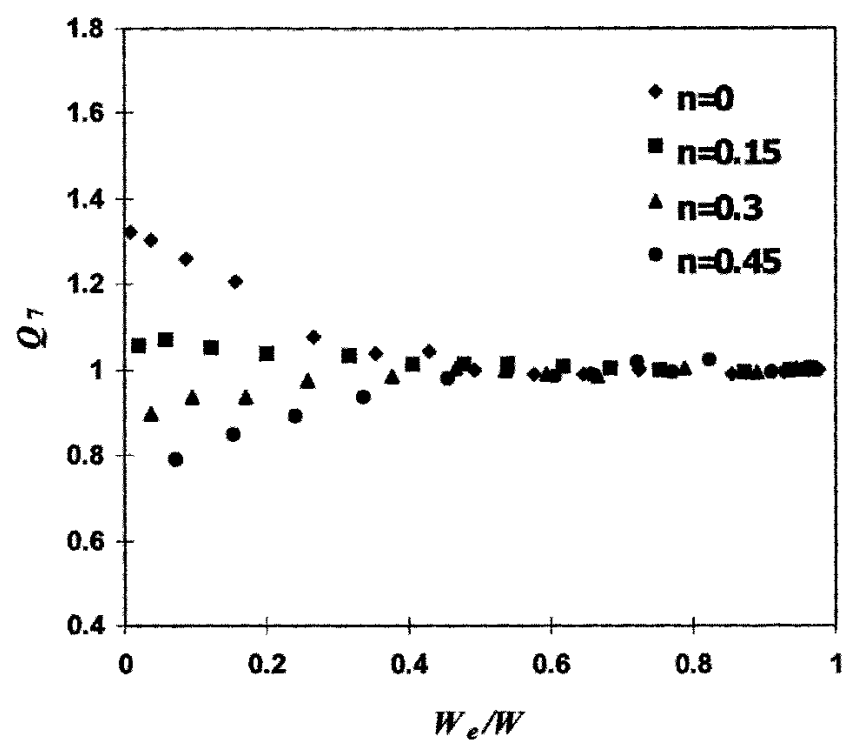

FIG. 10. Numerical results of function $\Gamma_{Q 7}\left(W_{\mathrm{e}} / W, n\right) \equiv H / H^{\mathrm{t}}$ obtained from our method.

conical indentation made on a linear elastic material. They interpreted the introduction of factor $\beta$ in Eq. (1) as a correction of Sneddon's solution. Assuming that the indenter was rigid and from simple geometry, an alternative expression of Eq. (1) was given as

$$
P_{\mathrm{m}}=\beta \frac{2}{\pi} \frac{E}{1-v^{2}} h_{\mathrm{m}}^{2} \tan \theta,
$$

which further led to

$$
H_{\mathrm{n}} / E_{\mathrm{r}}=\beta \frac{2}{\pi} \frac{\tan \theta}{24.5} .
$$

For the case concerned, they gave a $\beta$ of 1.065 , such that $H_{\mathrm{n}} / E_{\mathrm{r}}=0.07729$, which was slightly lower than our result of 0.07975 by not more than $3 \%$.

Dividing the right hand side of Eq. (8) by $E_{\mathrm{r}}^{\mathrm{t}}$, one obtains a dimensionless ratio $Q_{4} \equiv E_{\mathrm{r}} / E_{\mathrm{r}}^{\mathrm{t}}$, which can be written as

$$
Q_{4}=\left[H_{\mathrm{n}} / \Phi\left(W_{\mathrm{e}} / W\right)\right] / E_{\mathrm{r}}^{\mathrm{t}}=\Gamma_{Q^{4}}\left(W_{\mathrm{e}} / W, n\right) .
$$

Figure 7 shows the numerical results of $\Gamma_{Q 4}\left(W_{\mathrm{e}} / W, n\right)$ for different combinations of $W_{\mathrm{e}} / W$ and $n$, where the data points do not converge towards one in the low $W_{\mathrm{e}} / W$ 
region. We suggest that the functional relationship between $H_{\mathrm{n}} / E_{\mathrm{r}}$ and $W_{\mathrm{e}} / W$ [Eq. (31)] is still an approximate one but not accurate enough to reflect the details of all real cases. Unlike O\&P's and C\&C's methods, no correction scheme can be suggested to give further modification of the effectiveness of our method, while the error in estimating Young's modulus can still be controlled within $\pm 13 \%$.

We made use of the data reported by Alkorta et al. ${ }^{22}$ to examine the present results. The finite element results for estimating the reduced Young's modulus are given in Table I of their paper, where the $S / h_{\max }$ data should be divided by a factor of $10^{9}$ to convert them in the unit of $\mathrm{MPa}$. The ratios of $E_{\mathrm{r}}$ to the assigned $E_{\mathrm{r}}^{\mathrm{t}}$ derived from the three methods concerned are listed in Table I. We notice that the only data point obtained from our method falling outside the predicted error band is for the case of $\sigma_{\mathrm{y}} / E_{\mathrm{r}}=3.962 \times 10^{-5}$ and $n=0.4$ (i.e., $-17 \%$ ), whereas such conditions have exceeded far beyond the scope of the present discussion, i.e., $\sigma_{\mathrm{y}} / E_{r} \geqslant 4.55 \times 10^{-4}$ and $n \leqslant 0.45$. As such, the prediction of the error band of the method may no longer be valid.

\section{EVALUATION OF THE THREE METHODS USED FOR ESTIMATING HARDNESS}

Let $H^{\mathrm{t}}=P_{\mathrm{m}} / A\left(h_{\mathrm{cm}}{ }^{\mathrm{t}}\right)$ be the "true" hardness value. $Q_{5}$, $Q_{6}$ and $Q_{7}$ are obtained by dividing the right sides of Eqs. (2), (6), and (9) by $H^{\mathrm{t}}$, respectively:

$$
\begin{gathered}
Q_{5}=\left[P_{\mathrm{m}} / A\left(h_{\mathrm{cm}}\right)\right] / H^{\mathrm{t}}=\Gamma_{Q 5}\left(W_{\mathrm{e}} / W, n\right), \\
Q_{6}=\left[\frac{\pi}{4 \beta^{2}}\left[f\left(W_{\mathrm{e}} / W\right)\right]^{2} \frac{S_{\mathrm{u}}{ }^{2}}{P_{\mathrm{m}}}\right] / H^{\mathrm{t}}=\Gamma_{Q 6}\left(W_{\mathrm{e}} / W, n\right), \\
Q_{7}=\left\{\left[\frac{f\left(W_{\mathrm{e}} / W\right)}{\Phi\left(W_{\mathrm{e}} / W\right)}\right] H_{\mathrm{n}}\right\} / H^{\mathrm{t}}=\Gamma_{Q 7}\left(W_{\mathrm{e}} / W, n\right) .
\end{gathered}
$$

Figures 8-10 show the numerical results of these functions. The data points are diversified in the range of $W_{\mathrm{e}} / W<0.25$, where the error bands are much broader than those of the $E_{\mathrm{r}}$ data. Based on this observation, we decide not to introduce any correction scheme, since the accuracy of estimating hardness would not be further improved with this approach. The above results retain the general viewpoint that "hardness" should be regarded as a practical indicator rather than a fundamental property of a solid. However, hardness is so important for reflecting the elastic and plastic behaviors of a substance, so even though there is no unified measurement standard at present, but relative comparison of hardness values of different materials is still highly desired in many practical circumstances for material selection. One simple solution to satisfy this need is to refer to one single measurement method, such as O\&P's, because of its popularity in this area.

Finally, it should be pointed out that there have been new attempts in developing techniques for investigating accurate plastic properties of materials. ${ }^{23,24}$ One approach is to use two conical indenters of different halfincluded angles to perform depth-sensing indentation tests. Results may help to extract the yield stress and hardening behavior of the indented material. Obviously, understanding overall plastic behaviors should be more important than merely determining the hardness value. However, the related techniques are expected to be more complicated, and the validation of the methodology would require much more effort.

\section{CONCLUSIONS}

In this study, we evaluated the effectiveness of O\&P's method, C\&C's method, and a method recently developed by our group in determining Young's modulus and hardness based on instrumented indentation tests for the case of yield stress to reduced Young's modulus ratio $\geqslant 4.55 \times 10^{-4}$ and hardening coefficient $\leqslant 0.45$. Dimensional theorem and finite element simulations were conducted to produce standard reference results for this purpose. Results show that both O\&P's method and C\&C's method tend to overestimate the reduced Young's modulus in the range of $W_{\mathrm{e}} / W<0.25$. After introducing different correction functions in the forms of Eqs. (21) and (27), errors are suppressed to within $\pm 16 \%$. For our method, no correction procedure is recommended, while the error band is narrower, i.e., $\pm 13 \%$. For hardness measurements, the error bands of results deduced from all the three methods in the range of $W_{\mathrm{e}} / W<0.25$ are much broader than those of the Young's modulus measurements. Since the estimated hardness obtained in this range deviates so markedly from the "true" value, it appears to be less meaningful to introduce any artificial correction scheme for diminishing the errors. It is thus suggested that relative comparison of hardness data produced from the same standard technique is more preferred. O\&P's method is a good choice to serve for this purpose because of its popularity in this area of applications. It is finally noted that the ranges of error derived from the analysis could be different if different ranges of material parameters $\sigma_{\mathrm{y}} / E_{\mathrm{r}}$ and $n$ are considered.

\section{ACKNOWLEDGMENTS}

This work was supported by the National Natural Science Foundation of China (Grant Nos. 10432050 and 10372103), the Center for Smart Materials (Code: 1.A.310) of the Hong Kong Polytechnic University, and an internal grant (Code: G-T841) of the Hong Kong Polytechnic University. 


\section{REFERENCES}

1. Y-T. Cheng and C.M. Cheng: Scaling, dimension analysis, and indentation measurements. Mater. Sci. Eng. R 44, 91 (2004).

2. M.F. Doerner and W.D. Nix: A method for interpretating the data from depth-sensing indentation instruments. J. Mater. Res. 1, 601 (1986).

3. W.C. Oliver and G.M. Pharr: An improved technique for determining hardness and elastic modulus using load and displacement sensing indentation experiments. J. Mater. Res. 7, 1564 (1992).

4. G.M. Pharr, W.C. Oliver, and F.R. Brotzen: On the generality of the relationship among contact stiffness, contact area, and elastic modulus during indentation. J. Mater. Res. 7, 613 (1992).

5. W.C. Oliver and G.M. Pharr: Measurement of hardness and elastic modulus by instrumented indentation: Advances in understanding and refinements to methodology. J. Mater. Res. 19, 3 (2004).

6. Y-T. Cheng and C-M. Cheng: Relationships between hardness, elastic modulus, and the work of indentation. Appl. Phys. Lett. 73, 614 (1998).

7. Y-T. Cheng and Z. Li: Scaling relationships for indentation measurements. Philos. Mag. A 82, 1893 (2002).

8. Y-T. Cheng and Z. Li: Can stress-strain relationship be obtained from indentation curves using conical and pyramidal indenters? J. Mater. Res. 14, 3493 (1999).

9. Y-T. Cheng and Z. Li: Scaling relationships in conical indentation of elastic-perfectly plastic solids. Int. J. Solids Struct. 36, 1231 (1999).

10. Y-T. Cheng and Z. Li: What is indentation hardness? Surf. Coat. Technol. 133-134, 417 (2000).

11. D. Ma, C.W. Ong, and S.F. Wong: New relationship between Young's modulus and nonideally sharp indentation parameters. J. Mater. Res. 19, 2144 (2004).

12. D. Ma, C.W. Ong, S.F. Wong, and J. He: New method for determining Young's modulus by non-ideally sharp indentation. J. Mater. Res. 20, 1498 (2005).

13. D. Ma, C.W. Ong, J. Lu, and J. He: Determination of Young's modulus by nanoindentation. Sci. China Ser. E, Eng. Mater. Sci. 47, 398 (2004).

14. I.N. Sneddon: The relationship between load and penetration in the axisymmetric Bousinesq problem for a punch of arbitrary profile. Int. J. Eng. Sci. 3, 47 (1965).

15. ABAQUS Version 6.2 (Hibbitt, Karlsson \& Sorensen, Inc., Pawtucket, RI, 2001).

16. M. Dao, N. Chollacoop, K.J. Van Vliet, T.A. Venkatesh, and S. Suresh: Computational modeling of the forward and reverse problems in instrumented sharp indentation. Acta Mater. 49, 3899 (2001).

17. D. Ma, C.W. Ong, J. Lu, and J. He: Methodology for evaluation of yield strength and hardening behavior of metallic materials by indentation with spherical tip. J. Appl. Phys. 94, 288 (2003).

18. W. Ni and Y-T. Cheng: Modeling conical indentation in homogeneous materials and in hard films on soft substrates. J. Mater. Res. 20, 521 (2005).

19. Y-T. Cheng and C-M. Cheng: Effect of 'sinking in' and 'piling up' on estimating the contact area under load in indentation. Philos. Mag. Lett. 78, 115 (1998).

20. A. Bolshakov and G.M. Pharr: Influences of pileup on the measurement of mechanical properties by load and depth-sensing indentation techniques. J. Mater. Res. 13, 1049 (1998).

21. J.C. Hay, A. Bolshakov, and G.M. Pharr: A critical examination of the fundamental relations used in the analysis of nanoindentation data. J. Mater. Res. 14, 2296 (1999).

22. J. Alkorta, J.M. Martinez-Esnaola, and J. Gil Sevillano: Absence of one-to-one correspondence between elastoplastic properties and sharp-indentation load-penetration data. J. Mater. Res. 20, 432 (2005).

23. J.L. Bucaille, S. Stauss, E. Felder, and J. Michler: Determination of plastic properties of metals by instrumented indentation using different sharp indenters. Acta Mater. 51, 1663 (2003).

24. Y. Cao, X. Qian, J. Lu, and Z. Yao: An energy-based method to extract plastic properties of metal materials from conical indentation tests. J. Mater. Res. 20, 1194 (2005). 